Proceedings

\title{
Solid Dispersions as a Technological Strategy to Improve the Bio-Performance of Antiparasitic Drugs with Limited Solubility ${ }^{\dagger}$
}

\author{
Santiago N. Campos, Alicia G. Cid, Analía I. Romero, Mercedes Villegas, Cintia A. Briones Nieva, Elio E. Gonzo \\ and José M. Bermúdez *
}

Citation: Campos, S.N.; Cid, A.G.; Romero, A.I.; Villegas, M.; Briones Nieva, C.A.; Gonzo, E.E.; Bermúdez, J.M. Solid Dispersions as a Technological Strategy to Improve the Bio-Performance of Antiparasitic Drugs with Limited Solubility. Proceedings 2021, 78, 13. https:// doi.org/10.3390/IECP2020-08686

Published: 1 December 2020

Publisher's Note: MDPI stays neutral with regard to jurisdictional claims in published maps and institutional affiliations.

Copyright: (ㄷ) 2020 by the authors. Licensee MDPI, Basel, Switzerland. This article is an open access article distributed under the terms and conditions of the Creative Commons Attribution (CC BY) license (http://creativecommons.org/licenses/by/4.0/).
INIQUI (Instituto de Investigaciones para la Industria Química), UNSa (Universidad Nacional de Salta), CONICET (Consejo Nacional de Investigaciones Científicas y Técnicas), Salta 4400, Argentina; campossantiago93@gmail.com (S.N.C.); aliciagracielacid@gmail.com (A.G.C.); ailanarom@gmail.com (A.I.R.); mvillegas@unsa.edu.ar (M.V.); brionesncintia@gmail.com (C.A.B.N.); gonzo@unsa.edu.ar (E.E.G.)

* Correspondence: josemariabermudez@gmail.com; Tel.: +54-387-425-5410

† Presented at the 1st International Electronic Conference on Pharmaceutics, 1-15 December 2020; Available online: https://iecp2020.sciforum.net/.

\begin{abstract}
Albendazole (ABZ) and benznidazole (BZL) are drugs with low solubility used in parasitic infections treatment. In this research, solid dispersion (SD) technology was used to enhance ABZ and BZL performance by increasing their dissolution rate and solubility. SDs were prepared by the fusion method, employing Poloxamer 407 (P407) as carrier to disperse 32 of BZL or $50 \%$ w/w of ABZ. Furthermore, physical mixtures (PM) of P407 and either ABZ or BZL were also prepared, and then SDs and PMs were characterized. Dissolution tests of SDs, PMs and commercial formulations (CF) of $\mathrm{ABZ}$ and BZL were carried out and dissolution profiles were analyzed with the lumped mathematical model, which allowed parameters of pharmaceutical relevance to be obtained. The results indicated that ABZ SD presented an initial dissolution rate (IDR) 21-fold and 11-fold faster than PM and $\mathrm{CF}$, respectively, while the IDR of BZL SD was 2.5-fold and 4.5-fold faster than PM and CF, respectively. For BZL formulations, the time required to reach $80 \%$ dissolution of the drug (t80\%) was 4 (SD), 46 (PM), and $239 \mathrm{~min}(\mathrm{CF})$, while the dissolution efficiency (DE) at $30 \mathrm{~min}$ was 85 (DS), 71 (MF) and 65\% (FC). For ABZ formulations, t80\% was 2 (SD), value not reached (PM) and 40 min (CF), while the DE at 30 min was 85 (SD), 36 (MF) and 65\% (CF). The SDs developed notably increased the dissolution rate, in consonance with the values obtained from the pharmaceutical parameters, which could lead to faster absorption and, consequently, increase the bioavailability of these drugs.
\end{abstract}

Keywords: solid dispersions; benznidazole; albendazole; limited solubility; lumped model

\section{Introduction}

While albendazole (ABZ) is an antiparasitic drug used in both human and veterinary medicine for the treatment of parasitic infections [1], benznidazole (BZL) is a first-line drug against Chagas disease [2]. Nevertheless, they are both classified as class II drugs by the Biopharmaceutical Classification System (BCS) due to their low solubility, limiting their bioavailability [1,2].

The bioavailability of drugs is affected by several factors, such as drug solubility, permeability and dissolution rate. Drug molecules must be soluble enough in water to be delivered to the cells membrane and hydrophobic enough to pass through it [3]. Different strategies have been developed to overcome these issues, such as solid dispersion $[4,5]$, complexation with cyclodextrins [6-8], co-grinding [9], microcrystals [10-12], nanocrystals [12,13], and self-emulsifying formulation [14,15], among others. 
In 1961, Sekiguchi found that eutectic mixtures of poor soluble drugs and an inert soluble matrix enhanced drug dissolution rate and consequently its bioavailability [16]. Later, in 1971, Chiou and Riegelman defined the term solid dispersion (SD) as a dispersion of one or more active ingredients in a inert matrix or carrier in solid state [17].

Selection of carrier is essential, since this affects the dissolution rate, drug stability and other properties which have influence on manufacturing processes. Poloxamers are a family of amphiphilic triblock copolymers which consist of a central hydrophobic poly(propylene oxide) (PPO) molecule, which is flanked on both sides by two hydrophilic chains of poly(ethylene oxide) (PEO) [18,19]. The nature of the conforming blocks gives Poloxamers amphiphilic and surface active properties which vary depending on the $\mathrm{PPO} / \mathrm{PEO}$ ratio and molecular weight [18].

The aim of this work was to prepare and characterize ABZ and BZL SDs based on Poloxamer 407 (P407) as a polymeric carrier to enhance the solubility and dissolution rate of these drugs. Physico-chemical characterization and solubility studies were carried out and dissolution tests were performed to compare the dissolution behavior of SDs with physical mixtures (PMs) and commercial formulations (CFs). Drug dissolution data were analyzed by the lumped mathematical model and relevant pharmaceutical parameters were obtained, such as initial dissolution rate (IDR), dissolution efficiency (DE) and the time required to reach $80 \%$ dissolution of the drug $\left(\mathrm{t}_{80} \%\right)$.

\section{Materials and Methods}

\subsection{Materials}

CF of BZL (Abarax ${ }^{\circledR}$ Elesa) was kindly provided by Ministerio de Salud Pública de la Provincia de Salta (Salta, Argentina) and CF of ABZ was Vermizole (Ladefar Laboratory, Paraná, Argentina). Both were pulverized and sieved, and the 210- $\mu \mathrm{m}$ size fractions were used in the following assays. Powder BZL and ABZ were purchased form Sigma-Aldrich (St. Louis, MO, USA) and Todo Droga (Córdoba, Argentina), respectively. P407 was bought from BASF (Buenos Aires, Argentina). All other reagents were of analytical grade.

\subsection{BZL Extraction Procedure}

BZL extraction was carried out according to a procedure described by Garcia et al. [20]. Tablets were mashed and dissolved in ethanol by stirring at $45^{\circ} \mathrm{C}$. The obtained suspension was filtered, and BZL was recovered from the liquid phase by recrystallizing, adding ice-cold distilled water on an ice bath. The solid fraction was recovered by vacuum-filtration, and dried at $45{ }^{\circ} \mathrm{C}$ until constant weight. This solid was sieved, and 210$\mathrm{mm}$ BZL particles were stored protected from light at room temperature until use. The BZL obtained by this procedure was identified as extracted BZL (E-BZL).

\subsection{SDs Preparation}

Fusion method was used to prepare SDs. First, P407 was molten at $63{ }^{\circ} \mathrm{C}$ and loaded with $32 \% w / w$ BZL or $50 \% w / w$ ABZ by stirring, respectively. The preparations were rapidly cooled with liquid nitrogen and pulverized and the $210-\mu \mathrm{m}$ size fractions were stored in screw-cap vials until use. PMs were prepared by mixing the sifted $210-\mu \mathrm{m}$ size fraction of the components in same proportion as the SDs. PMs were also kept in screw-cap vials until use.

\subsection{Physico-Chemical Carachterization}

\subsubsection{X-ray Diffraction}

CFs, PMs and SDs of BZL and ABZ were characterized by X-ray diffraction (XRD) using a DRX Philips PW1800 diffractometer (Philips, Almelo, The Netherlands). Assays were performed at $40 \mathrm{KV}$ and $30 \mathrm{~mA}$ in a range of $5-70^{\circ} 2 \theta$ at a rate of $0.02^{\circ} 2 \theta / \mathrm{s}$. 


\subsubsection{Scanning Electron Microscopy}

Sample morphology was observed with a scanning electron microscope JEOL JSM6480LV-Japan, in the Laboratory of Scanning Electron Microscopy (LASEM, ANPCyTUNSa-CONICET). Previously, samples were gold coated using a Denton Vacuum metallizer, LLC, Moorestown, NJ, USA, Desk-IV.

\subsubsection{Phase and Saturation Solubility Studies}

For phase solubility studies, an excess of ABZ or BZL was added to sealed glass vials containing $5 \mathrm{~mL}$ of $0.1 \mathrm{~N} \mathrm{HCl}$ with increasing concentration of P407. Vials were shaken in a bath at room temperature in order to reach solubility equilibrium. After $96 \mathrm{~h}$, suspensions were filtered, diluted and spectrophotometrcally analyzed at $\lambda=324 \mathrm{~nm}$ (BZL) or $\lambda$ $=302 \mathrm{~nm}$ (ABZ). Concentrations were calculated using the corresponding calibration curve, constructed with standard solutions of each drug in $0.1 \mathrm{HCl}$.

For saturation solubility assays, an excess amount of SDs and PMs of each drug was added to sealed glass vials containing $10 \mathrm{~mL}$ of $0.1 \mathrm{~N} \mathrm{HCl}$. Suspensions were continuously stirred in a water bath at $37^{\circ} \mathrm{C}$ for 4 days. After that, samples were filtered, and filtrates were suitably diluted and analyzed spectrophotometrically to determine drug concentration.

\subsection{Dissolution Test}

Dissolution tests of CFs, PMs and SDs were performed in an USPXXIV dissolution apparatus 2 (SOTAX AT 7 smart). The necessary amount of each sample was weighed to add the equivalent of $100 \mathrm{mg}$ of BZL or $50 \mathrm{mg}$ of $\mathrm{ABZ}$ into $900 \mathrm{~mL}$ of filtered and degassed $0.1 \mathrm{~N} \mathrm{HCl}$. The experiments were performed at $37 \pm 0.5{ }^{\circ} \mathrm{C}$ with the rotational paddle speed set at $50 \mathrm{rpm}$. Aliquots of $4 \mathrm{~mL}$ were withdrawn in specific intervals, adding the same amount of fresh medium to keep volume constant. Samples were filtered and accurately diluted, and concentration of dissolved drug was measured spectrophotometrically at $324 \mathrm{~nm}$ (BZL) or $302 \mathrm{~nm}$ (ABZ). Each dissolution assay was performed in triplicate.

Dissolution data were analyzed by a lumped second-order kinetic model developed and validated by our research group [21,22]. Different parameters were calculated to compare the different dissolution profiles: initial dissolution rate (IDR), dissolution efficiency (DE), and dissolution time ( $\left(\mathrm{t}^{\circ} \%\right)$, according to the independent statistical analysis methods [23].

\section{Results}

\subsection{Physical-Chemical Carachterization}

\subsubsection{X-ray Diffraction}

To determine the crystalline state of materials, diffractograms of E-BZL, P-ABZ, PMs and SDs were obtained as shown in Figure 1. Several peaks in the diffraction profiles indicated that E-BZL and P-ABZ were in crystalline state, with characteristic peaks at 7.41, 10.9, 16,9, 22, 23.7, and 25.4 ${ }^{\circ}$, and 6.80, 11.30, 13.8, 17.9, 19.5, 20.8, 22.1, 24.43, 24.6, 27.2, 28.4 and $29.9^{\circ}$ for E-BZL and P-ABZ, respectively. Diffractograms also showed peaks for P407 at 19.2 and $23.3^{\circ}$, as reported in [24]. SD and PM spectra indicated that some peaks of both drugs were attenuated. 


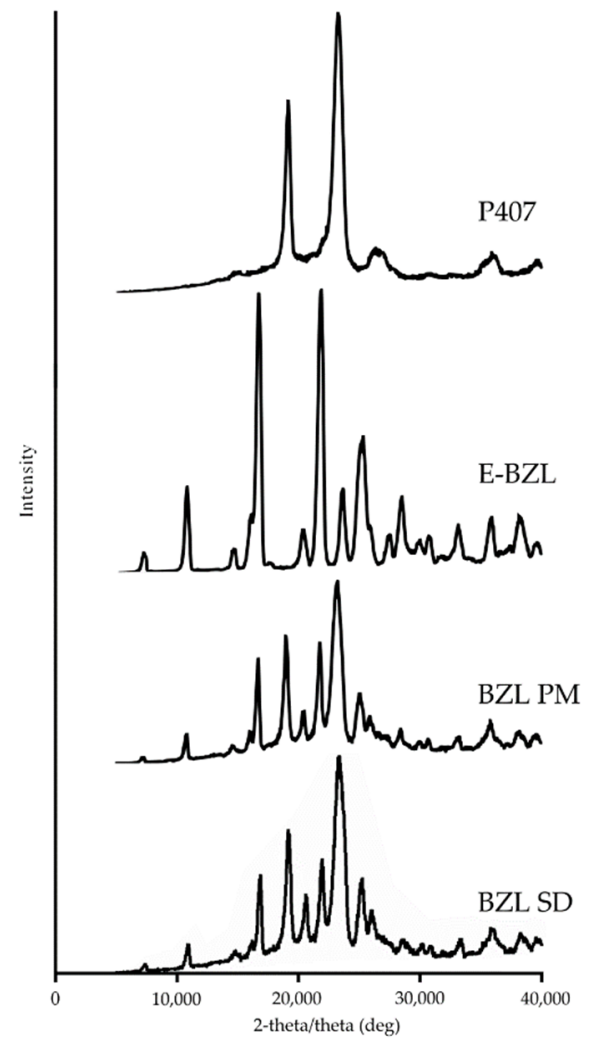

(a)

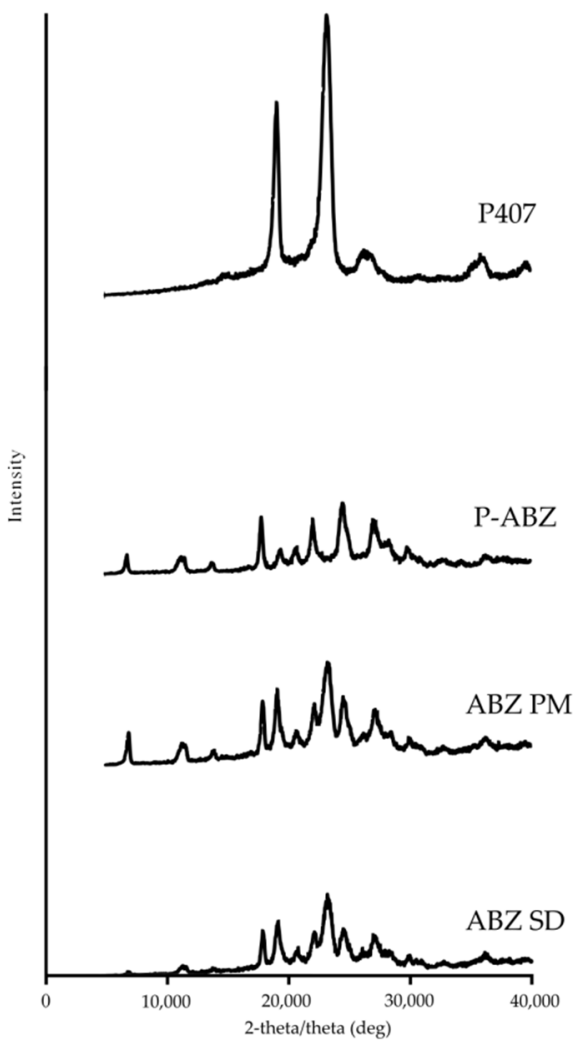

(b)

Figure 1. XDR diffractograms: (a) BZL formulations, (b) ABZ formulations.

\subsubsection{Scanning Electron Microscopy}

SEM (Figure 2) was used to characterize the morphology of P-ABZ, E-BZL, P407, DSs, PMs of both ABZ and BZL. Micrographs showed needle-shaped crystals of P-ABZ with different sizes, whereas E-BZL presented as particles of irregular shape and rough surface. P407 appeared as spherical particles with a smooth surface and irregular shape. Images of SDs showed particles of different sizes and irregular surface could be observed. In PM, polymer and drugs, which were dispersed in P407, conserved their own structure.

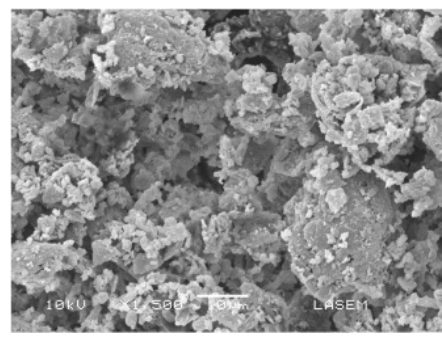

(a)

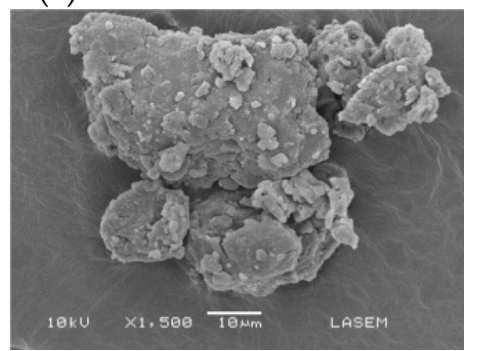

(e)

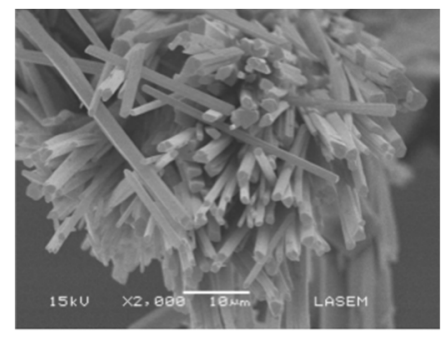

(b)

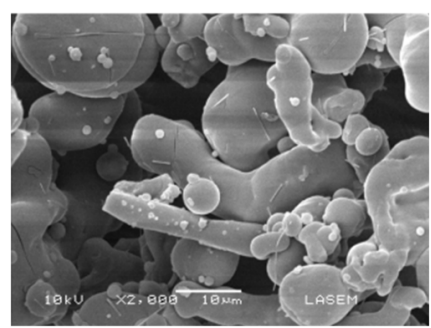

(c)

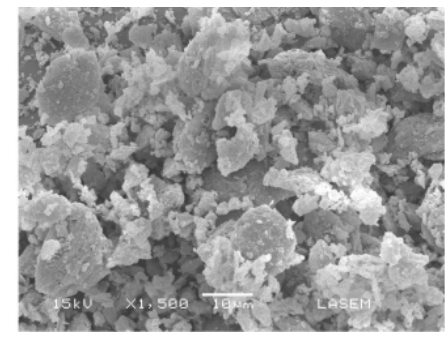

(d)

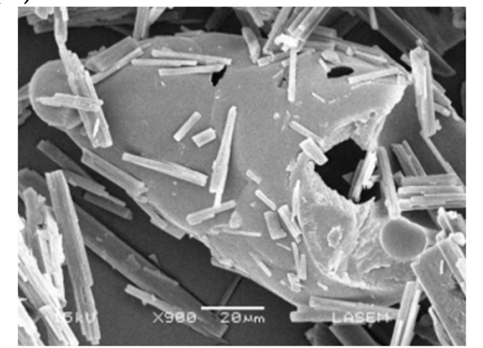

(f)

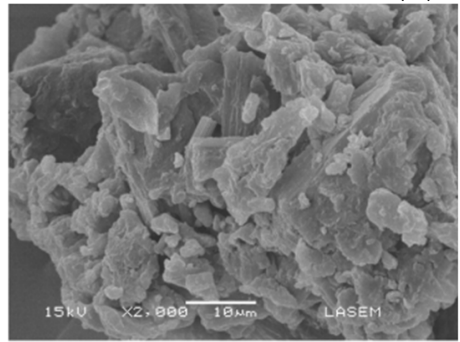

(g)

Figure 2. Scanning electron micrographs: (a) P-ABZ, (b) E-BZL, (c) P407, (d) ABZ PM, (e) ABZ SD, (f), BZL PM, (g) BZL SD. 


\subsubsection{Phase and Saturation Solubility Studies}

Phase solubility assay results indicated a linear increase in solubility with P407 concentration, both for ABZ and for BZL. In the case of ABZ, a nearly two-fold increase in solubility was observed, while this value was nearly 1.5 for BZL, for an increasing concentration of $\mathrm{P} 407$ from $5 \%$ to $15 \% w / v$. ABZ and BZL solubility were estimated by extrapolating the line equation to $0 \% w / w P 407$, finding values of $0.320 \mathrm{mg} / \mathrm{mL}$ and $0.265 \mathrm{mg} / \mathrm{mL}$, respectively.

The saturation solubility of $\mathrm{ABZ}$ was found to be $0.801 \pm 0.032 \mathrm{mg} / \mathrm{mL}$ and $0.832 \pm$ $0.040 \mathrm{mg} / \mathrm{mL}$ for SD and PM, respectively. In the case of BZL, solubility was $0.260 \pm 0.001$ $\mathrm{mg} / \mathrm{mL}$ and $0.274 \pm 0.010 \mathrm{mg} / \mathrm{mL}$ for SD and PM, respectively.

\subsection{Dissolution Test}

Since the dissolution rate of drugs is related to its bioperformance [3], dissolution test of SDs, PMs and CFs were carried out.

Dissolution profiles are shown in Figure 3 and experimental data were fitted using a lumped model, which considers a second-order kinetic (Equation (1)):

$$
\mathrm{M}_{\%}=\frac{\mathrm{a} \times \mathrm{t}}{1+\mathrm{b} \times \mathrm{t}}
$$

where $\mathrm{M} \%$ is the cumulative percentage of drug released at time $t$, and a and $b$ are model parameters given in $\%^{*} \mathrm{~min}^{-1}$ and $\mathrm{min}^{-1}$, respectively.

This model makes it possible to calculate the initial dissolution rate (IDR), as shown in Equation (2):

$$
\operatorname{IDR}=\left.\frac{\mathrm{dM}_{\%}}{\mathrm{dt}}\right|_{\mathrm{t}=0}=\mathrm{a}
$$

Results for IDR indicated that SDs of ABZ presented values 21-fold and 11-fold faster than PM and CF, respectively, while the IDR of BZL SD was 2.5-fold and 4.5-fold faster than $\mathrm{PM}$ and $\mathrm{CF}$, respectively.

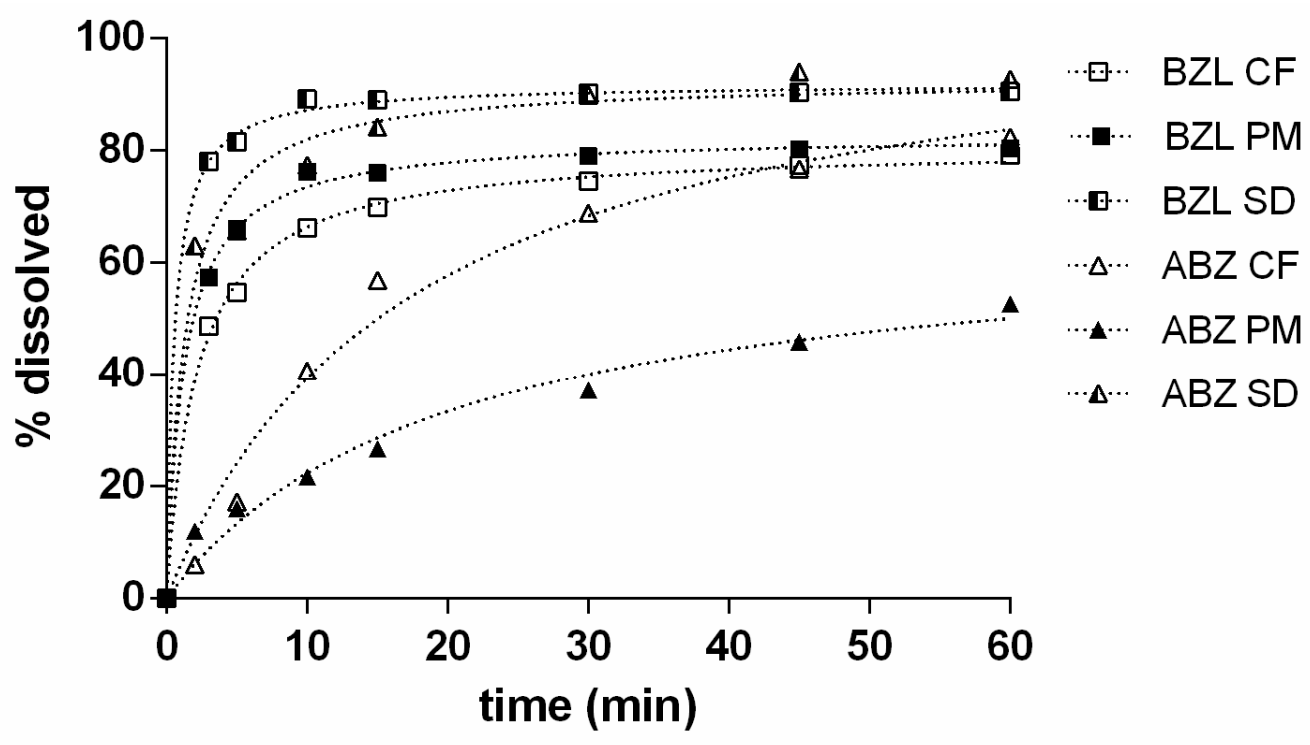

Figure 3. Dissolution profiles of different formulations of ABZ and BZL. Symbols are the mean value of experimental data obtained by triplicate and dotted lines represent the prediction of released profile from lumped model fit. Error bars were omitted for clarity purposes.

Dissolution efficiency (DE) and t80\% are other parameters used to assess formulation behavior. $\mathrm{DE}$ is defined as the area under the dissolution profile up to a certain final time 
$\left(\mathrm{t}_{\mathrm{F}}\right)$, expressed as the percentage of the rectangular area described by $100 \%$ dissolution at the same $t_{\mathrm{F}}$ [23]. DE was calculated from Equation (3), considering Equation (1):

$$
\mathrm{DE}=\frac{\int_{0}^{\mathrm{t}_{\mathrm{F}}} \mathrm{X} \% \mathrm{dt}}{100 \times \mathrm{t}_{\mathrm{F}}} \times 100=\left(\frac{\mathrm{a}}{\mathrm{b}^{2}}\right) \frac{\left[\mathrm{b} \times \mathrm{t}_{\mathrm{F}}-\ln \left(1+\mathrm{b} \times \mathrm{t}_{\mathrm{F}}\right)\right]}{100 \times \mathrm{t}_{\mathrm{F}}} \times 100
$$

Therefore, $\mathrm{DE}=100 \%$ indicates an instantaneous dissolution of the drug.

The value of $\mathrm{t}_{80} \%$ represents the time required to reach $80 \%$ dissolution of the drug and can be obtained from Equation (4) for $\mathrm{X} \%=80 \%$.

$$
\mathrm{t}_{\mathrm{X} \%}=\frac{\mathrm{X} \%}{\mathrm{a}-\mathrm{b} \times \mathrm{X} \%}
$$

The results for $\mathrm{t} 80 \%$, DE and IDR for the different formulations of BZL and ABZ are shown in Table 1.

Table 1. Parameters of pharmaceutical relevance obtained from the mathematical model.

\begin{tabular}{cccc}
\hline Sample & IDR $\left(\mathbf{\%}^{*} \mathbf{m i n}^{-\mathbf{1}}\right)$ & $\mathbf{t 8 0 \%} \mathbf{( m i n )}$ & DE (\%) \\
\hline ABZ CF & 6.74 & 40.31 & 65.11 \\
ABZ PM & 3.34 & NR & 35.93 \\
ABZ SD & 71.62 & 1.69 & 84.91 \\
BZL CF & 37.24 & 239.21 & 64.98 \\
BZL PM & 66.03 & 46.45 & 71.58 \\
BZL SD & 170.23 & 3.64 & 85.18 \\
\hline
\end{tabular}

DE: dissolution efficiency at $30 \mathrm{~min}, \mathrm{NR}$ : not reached.

\section{Discussion}

\subsection{Physico-Chemical Carachterization}

\subsubsection{X-ray Diffraction}

The peak pattern of PMs and SDs presented no modifications in P407 and drug signals, indicating the absence of interactions between the polymer and ABZ or BZL. On the other hand, these signals were attenuated, which suggests a partial lost in crystallinity, leading probably to an increase in the solubility, since the amorphous state is more soluble than the crystalline one [8].

\subsubsection{Scanning Electron Microscopy}

In the micrographs, SDs appeared as particles with different sizes, in which the original morphology of both components could not be distinguished, indicating that $\mathrm{ABZ}$ and BZL could be dissolved within the polymer.

\subsubsection{Phase and Saturation Solubility}

As mentioned before, the solubility of ABZ and BZL increased linearly with P407 concentration. This could be due the formation of polymer micelles with a hydrophobic core in which drug molecules can be incorporated, avoiding contact with the aqueous medium that promotes drug dissolution.

\subsection{Dissolution Test}

Analyzing the obtained parameters, IDR was several times higher for SDs and PMs. This indicates that P407 is capable of increasing not only the solubility of both ABZ and BZL, but also the IDR. This is important, because dissolution rate would play an essential role in drugs bioavailability.

DE values near $85 \%$ for SD of both BZL and ABZ indicate good performance, since DE considers the amount of drug dissolved and the dissolution rate [25]. On the other hand, t80\% is considered acceptable for immediate release when it is lower than $45 \mathrm{~min}$ [23]. 
Thus, SDs seem to be the better option among the studied formulations, since they have the best dissolution behavior.

\section{Conclusions}

SDs using P407 as polymeric carrier seem to be an effective alternative for immediate drug release. This technology allowed an improvement in ABZ and BZL solubility and dissolution rate compared to CFs and PMs, which could result in an enhancement in their absorption and bioavailability.

\section{Institutional Review Board Statement: Not applicable.}

Informed Consent Statement: Not applicable.

Data Availability Statement: The data presented in this study are available on request from the corresponding author.

\section{References}

1. Dayan, A.D. Albendazole, mebendazole and praziquantel. Review of non-clinical toxicity and pharmacokinetics. Acta Trop. 2003, 86, 141-159, doi:10.1016/s0001-706x(03)00031-7.

2. Bahia, M.T.; Diniz Lde, F.; Mosqueira, V.C. Therapeutical approaches under investigation for treatment of Chagas disease. $E x$ pert Opin. Investig. Drugs 2014, 23, 1225-1237, doi:10.1517/13543784.2014.922952.

3. Krishnaiah, Y. Pharmaceutical Technologies for Enhancing Oral Bioavailability of Poorly Soluble Drugs. J. Bioequiv. Bioavailab. 2010, 2, doi:10.4172/jbb.1000027.

4. Castro, S.G.; Bruni, S.S.; Lanusse, C.E.; Allemandi, D.A.; Palma, S.D. Improved Albendazole Dissolution Rate in Pluronic 188 Solid Dispersions. AAPS PharmSciTech 2010, 11, 1518-1525, doi:10.1208/s12249-010-9517-6.

5. Lima, Á.A.N.; Soares-Sobrinho, J.L.; Silva, J.L.; Corrêa-Júnior, R.A.C.; Lyra, M.A.M.; Santos, F.L.A.; Oliveira, B.G.; Hernandes, M.Z.; Rolim, L.A.; Rolim-Neto, P.J. The Use of Solid Dispersion Systems in Hydrophilic Carriers to Increase Benznidazole Solubility. J. Pharm. Sci. 2011, 100, 2443-2451, doi:10.1002/jps.22436.

6. García, A.; Leonardi, D.; Lamas, M.C. Promising applications in drug delivery systems of a novel $\beta$-cyclodextrin derivative obtained by green synthesis. Bioorg. Med. Chem. 2016, 26, 602-608, doi:10.1016/j.bmcl.2015.11.067.

7. Soares-Sobrinho, J.L.; Santos, F.L.A.; Lyra, M.A.M.; Alves, L.D.S.; Rolim, L.A.; Lima, A.A.N.; Nunes, L.C.C.; Soares, M.F.R.; Rolim-Neto, P.J.; Torres-Labandeira, J.J. Benznidazole drug delivery by binary and multicomponent inclusion complexes using cyclodextrins and polymers. Carbohydr. Polym. 2012, 89, 323-330, doi:10.1016/j.carbpol.2012.02.042.

8. Sobrinho, J.L.S.; Soares, M.F.; Labandeira, J.J.T.; Alves, L.D.S.; Rolim Neto, P.J. Improving the solubility of the antichagasic drug benznidazole through formation of inclusion complexes with cyclodextrins. Quim. Nova 2011, 34, 1534-1538.

9. Vogt, M.; Kunath, K.; Dressman, J.B. Dissolution improvement of four poorly water soluble drugs by cogrinding with commonly used excipients. Eur. J. Pharm Biopharm. 2008, 68, 330-337, doi:10.1016/j.ejpb.2007.05.009.

10. Priotti, J.; Codina, A.V.; Leonardi, D.; Vasconi, M.D.; Hinrichsen, L.I.; Lamas, M.C. Albendazole Microcrystal Formulations Based on Chitosan and Cellulose Derivatives: Physicochemical Characterization and In Vitro Parasiticidal Activity in Trichinella spiralis Adult Worms. AAPS PharmSciTech 2017, 18, 947-956, doi:10.1208/s12249-016-0659-z.

11. Maximiano, F.P.; de Paula, L.M.; Figueiredo, V.P.; de Andrade, I.M.; Talvani, A.; Sá-Barreto, L.C.; Bahia, M.T.; Cunha-Filho, M.S.S. Benznidazole microcrystal preparation by solvent change precipitation and in vivo evaluation in the treatment of Chagas disease. Eur. J. Pharm. Biopharm. 2011, 78, 377-384, doi:10.1016/j.ejpb.2011.03.003.

12. Seremeta, K.P.; Arrúa, E.C.; Okulik, N.B.; Salomon, C.J. Development and characterization of benznidazole nano- and microparticles: A new tool for pediatric treatment of Chagas disease? Colloid Surf. B 2019, 177, 169-177, doi:10.1016/j.colsurfb.2019.01.039.

13. Paredes, A.J.; Llabot, J.M.; Sánchez Bruni, S.; Allemandi, D.; Palma, S.D. Self-dispersible nanocrystals of albendazole produced by high pressure homogenization and spray-drying. Drug Dev. Ind. Pharm. 2016, 42, 1564-1570, doi:10.3109/03639045.2016.1151036.

14. Mukherjee, T.; Plakogiannis, F.M. Development and oral bioavailability assessment of a supersaturated self-microemulsifying drug delivery system (SMEDDS) of albendazole. J. Pharm. Pharmacol. 2010, 62, 1112-1120, doi:10.1111/j.2042-7158.2010.01149.x.

15. Mazzeti, A.L.; Oliveira, L.T.; Gonçalves, K.R.; Schaun, G.C.; Mosqueira, V.C.F.; Bahia, M.T. Benznidazole self-emulsifying delivery system: A novel alternative dosage form for Chagas disease treatment. Eur. J. Pharm. Sci. 2020, 145, 105234, doi:10.1016/j.ejps.2020.105234.

16. Sekiguchi, K.; Obi, N. Studies on Absorption of Eutectic Mixture. I. A Comparison of the Behavior of Eutectic Mixture of Sulfathiazole and that of Ordinary Sulfathiazole in Man. Chem. Pharm. Bull. 1961, 9, 866-872.

17. Chiou, W.L.; Riegelman, S. Pharmaceutical applications of solid dispersion systems. J. Pharm. Sci. 1971, 60, 1281-1302.

18. Bodratti, A.M.; Alexandridis, P. Formulation of Poloxamers for Drug Delivery. J. Funct. Biomater. 2018, 9, doi:10.3390/jfb9010011. 
19. Moghimi, S.M.; Hunter, A.C. Poloxamers and poloxamines in nanoparticle engineering and experimental medicine. Trends Biotechnol. 2000, 18, 412-420, doi:10.1016/S0167-7799(00)01485-2.

20. García, M.C.; Ponce, N.E.; Sanmarco, L.M.; Manzo, R.H.; Jimenez-Kairuz, A.F.; Aoki, M.P. Clomipramine and Benznidazole Act Synergistically and Ameliorate the Outcome of Experimental Chagas Disease. Antimicrob. Agents Chemother. 2016, 60, 3700-3708, doi:10.1128/aac.00404-16.

21. Fernández-Colino, A.; Bermudez, J.M.; Arias, F.; Quinteros, D.; Gonzo, E. Development of a mechanism and an accurate and simple mathematical model for the description of drug release: Application to a relevant example of acetazolamide-controlled release from a bio-inspired elastin-based hydrogel. Mat. Sci. Eng. C Mater. 2016, 61, 286-292.

22. Romero, A.I.; Villegas, M.; Cid, A.G.; Parentis, M.L.; Gonzo, E.E.; Bermúdez, J.M. Validation of kinetic modeling of progesterone release from polymeric membranes. Asian J. Pharm. Sci. 2018, 13, 54-62.

23. Costa, P.; Sousa Lobo, J.M. Modeling and comparison of dissolution profiles. Eur. J. Pharm. Sci. 2001, 13, 123-133, doi:10.1016/S0928-0987(01)00095-1.

24. El-Badry, M.; Hassan, M.A.; Ibrahim, M.A.; Elsaghir, H. Performance of poloxamer 407 as hydrophilic carrier on the binary mixtures with nimesulide. Farmacia 2013, 61, 1137-1150.

25. Simonazzi, A.; Davies, C.; Cid, A.G.; Gonzo, E.; Parada, L.; Bermúdez, J.M. Preparation and characterization of Poloxamer 407 solid dispersions as an alternative strategy to improve benznidazole bioperformance. J. Pharm. Sci. 2018, 107, 2829-2836. 